\title{
Accelerated Ageing for Prediction of Storability in Onion (Allium cepa L.) Seeds
}

\author{
Manjunath V. Balikai ${ }^{1 *}$, N. K. Biradarpatil ${ }^{1}$, Jagadish Hosamani ${ }^{2}$ and M. S. Biradar ${ }^{2}$ \\ ${ }^{1}$ Department of Seed Science and Technology, ${ }^{2}$ Department of Horticulture, College of \\ Agriculture, Vijayapura University of Agricultural Sciences, Dharwad-580005, \\ Karnataka, India \\ *Corresponding author
}

A B S T R A C T

Keywords

Germination, vigour, accelerated ageing, onion

Article Info

Accepted:

25 February 2020

Available Online:

10 March 2020
A laboratory experiment was conducted to predict the storability by accelerated ageing in onion in the Department of Seed Science and Technology, University of Agricultural Sciences, Dharwad, during 201819. The experiment consisted of fifteen seed lots with varied levels of germination and it was laid out in CRD design with four replications. All seed lots were subjected to: standard germination test, electrical conductivity test, radicle emergence test, accelerated ageing test and field emergence test. The results indicated that, accelerated ageing (AA) and field emergence was highly correlated with $\mathrm{r}=0.925$ and also with germination $(r=0.923)$. The accelerated ageing and electrical conductivity was correlated with $r=-0.933$. The correlation between accelerated ageing and mean germination time was -0.857 . Thus, AA test can be potentially used for predicting the storability and evaluating seed vigour in onion seeds.

\section{Introduction}

Onion (Allium cepa L.) is one of the commercially important vegetables and major among the bulbous crops belongs to the family Amaryllidaceae. Currently, the demand for onion is increasing, mainly due to its nutritive and medicinal value such as, antibacterial and antioxidant effects (Doijode, 2001). The non availability of quality seeds coupled with poor germination and low seedling vigour are the serious problems limiting the production of onion. Further, onion seeds exhibit poor storability because of physio-chemical factors like temperature, moisture content, atmospheric relative humidity, initial seed quality, the physical and chemical composition of seed, storage structure, gaseous exchange, packaging materials etc. (Doijode, 1998). The use of physiological low 
quality seeds is a common practice under tropical and sub-tropical conditions leading to inadequate plant population in the field. Further the carry over seeds generally exhibit poor germinability, less vigour and decline in their ability to germinate and emerge into vigorous seedlings leading are the problems associated with successful crop production. Therefore, a reliable estimation of seed vigour is of prime importance to determine the planting value of the seed, particularly for those that have poor storability like onion (Yousof et al., 2016).

Different types of vigour tests assess either directly or indirectly, the potential performance of the seed lot and provide more sensitive information of a seed lot or between seed lots than does the standard germination test. The extensive use of vigour assessments in quality control programme of the seed industry is encouraging (McDonald, 1995). Accelerated ageing test in one of the vigour test which is carried out to predict the relative performance and storability of seeds. It is necessary to establish relationship between germination before and after accelerated ageing for onion and anticipating the storage period through this vigour test for onion seeds. With this background, the current investigation was carried out to assess the suitability of accelerated ageing test for predicting the storage potentiality of seed lots.

\section{Materials and Methods}

Fifteen seed lots of onion crop having varied germination percentages were selected. The fresh seed lots of Arka kalyan variety were collected from Seed Unit, UAS, Dharwad. The seeds were subjected to accelerated ageing test and natural ageing at Seed Quality Research Laboratory, National Seed Project, University of Agricultural Sciences, Dharwad. The experiment was laid out in CRD (Completely Randomised Design) with four replications.
The mean data obtained from the experimentation was statistically analysed and subjected to the analysis of variance by adopting appropriate statistical methods as outlined by Panse and Sukhatme (1985) and Sundararaj et al., (1972).

Germination test was conducted as per the procedure recommended by International Seed Testing Association (Anon., 2013). Accelerated ageing test was conducted by distributing seeds uniformly on a single layer of wire mesh tray fitted in plastic boxes having $40 \mathrm{ml}$ of distilled water at the bottom. The boxes were placed in accelerated ageing chamber after closing their lids. The seeds were aged at $40 \pm 1{ }^{\circ} \mathrm{C}$ temperature and about 95 per cent relative humidity for $48 \mathrm{~h}$ and then tested for germination by following the procedure given by Delouche and Baskin, (1973). The number of normal seedlings were counted and expressed in per cent.

\section{Results and Discussion}

The results of the study indicated that, the seed lots with varied level of germination. The lots exhibited variations in vigour levels also and this variation was not associated with germination. Among the seed lots, lot 13 recorded higher germination of 92.00 and 81.75 per cent before and after accelerated ageing, followed by lot 11 which recorded 89.00 and 69.50 per cent, before and after accelerated ageing, respectively (Table 1). These seed lots were kept under ambient conditions to correlate the germination percentages for accelerated ageing. The lot 13 recorded germination of 81.75 per cent (Table 2) at the end of seventh month of storage which was correlated with the germination after accelerated ageing. Similarly, lot 11 recorded germination of 69.00 per cent at the end of sixth month of storage correlating to germination after accelerated ageing. Lot 14 with lower initial germination of 40.00 per 
cent almost lost its germination after accelerated ageing which recorded 1.50 per cent germination and it was correlating with germination after accelerated ageing at fifth of storage period (Fig. 1). Similarly, lot 15 which recorded germination of 58.00 and 26.75 per cent before and after accelerated ageing, which was correlated with germination after accelerated ageing at the end of fifth month of storage period (Fig. 1).

The germination after accelerated ageing showed significant correlation $(r=0.955)$ with seedling vigour index, field emergence $(r=$ $0.925)$ and before germination $(r=0.923)$ (Table 3). Among the other tests the correlation of electrical conductivity was found to be negative with accelerated ageing $(r=-0.933)$, field emergence $(r=-0.906)$ and positive with mean germination time $(\mathrm{r}=$ 0.872), followed by accelerated ageing which was positively correlated with field emergence $(\mathrm{r}=0.925)$ and negatively correlated with mean germination time $(\mathrm{r}=-0.857)$. The mean germination time also negatively correlated with field emergence $(r=-0.900)$ (Table 3$)$.

The coefficient of determination (Fig. 2) between germination after accelerated ageing and germination before gave a significant positive value $\mathrm{R}^{2}=0.922$. The regression relationship between germination after accelerated ageing and field emergence for seed lots show a positive relationship with $\mathrm{R}^{2}$ $=0.910$, thus indicating 91 per cent of variation in field emergence was contributed by germination after accelerated ageing (Fig. 3). However, germination after accelerated ageing contributed 86.2 per cent variation to electrical conductivity indicating negative relationship (Fig. 4). The germination after accelerated ageing showed significantly negative relation with a variation of 73.7 per cent contributed to mean germination time (Fig. 5). The reduction in germination after accelerated ageing might be due to the stress conditions (high temperature and high relative humidity) which resulted in enhanced ageing conditions reducing the effects of internal factors that inhibit the germination process under normal condition (Khan et al., 2003). In the present study lot 13 and lot 11 correlated to germination after accelerated ageing at the end of seventh month of storage accounting for higher initial seed quality. Similarly, lot 14 and lot 15 correlated to germination after accelerated ageing at the end of fifth month of storage accounting for lower initial seed quality. As natural ageing is time consuming, the storage potential can be assessed earlier without waiting for natural ageing by accelerated ageing. The results are in line with Maskri et al., (2004) in watermelon. The reduction in germination and vigour is attributed to DNA degradation which leads to impaired transcription causing imperfect or defective enzyme synthesis which is essential for earlier stages of germination. The decreases in seed germination by accelerated ageing might be a result of progressive loss of seed viability due to ageing and could be used as an excellent method for determination of vigour (Ashraf and Habib, 2011; Mosavi et al., 2011).

From the results it could be concluded that, accelerated ageing test was able to determine true potential of lots and achieved the assessment of vigour and storability of onion seeds. However, repeatability and reproducibility of this vigour test need to be assessed through different laboratories. 
Table.1 Effect of accelerated ageing on germination in onion seed

\begin{tabular}{|c|c|c|}
\hline Seed lots & $\begin{array}{c}\text { Germination before } \\
\text { accelerated ageing }(\mathbf{\%})\end{array}$ & $\begin{array}{c}\text { Germination after accelerated } \\
\text { ageing }(\mathbf{\%})\end{array}$ \\
\hline $\mathrm{L}_{1}$ & $83.00(65.65)$ & $59.50(50.37)$ \\
\hline $\mathrm{L}_{2}$ & $45.00(42.11)$ & $13.75(21.68)$ \\
\hline $\mathrm{L}_{3}$ & $80.00(63.48)$ & $56.75(48.81)$ \\
\hline $\mathrm{L}_{4}$ & $62.00(51.94)$ & $44.75(41.92)$ \\
\hline $\mathrm{L}_{5}$ & $50.00(44.98)$ & $11.75(19.95)$ \\
\hline $\mathrm{L}_{6}$ & $71.00(57.40)$ & $48.75(44.22)$ \\
\hline $\mathrm{L}_{7}$ & $68.00(55.53)$ & $37.00(37.43)$ \\
\hline $\mathrm{L}_{8}$ & $65.00(53.73)$ & $44.00(41.92)$ \\
\hline $\mathrm{L}_{9}$ & $77.00(61.32)$ & $50.50(45.17)$ \\
\hline $\mathrm{L}_{10}$ & $74.00(59.36)$ & $48.50(44.03)$ \\
\hline $\mathrm{L}_{1}$ & $89.00(70.81)$ & $69.50(49.20)$ \\
\hline $\mathrm{L}_{12}$ & $86.00(68.03)$ & $58.75(49.98)$ \\
\hline $\mathrm{L}_{13}$ & $92.00(73.75)$ & $81.75(54.72)$ \\
\hline $\mathrm{L}_{14}$ & $40.00(39.21)$ & $1.50(6.53)$ \\
\hline $\mathrm{L}_{15}$ & $58.00(49.59)$ & $26.75(31.07)$ \\
\hline $\mathrm{Mean}$ & $\mathbf{6 9 . 5 0}(\mathbf{5 7 . 1 2})$ & $\mathbf{3 9 . 1 1}$ \\
\hline $\mathrm{SE}_{4} \pm$ & $\mathbf{1 . 2 1}$ & $\mathbf{0 . 8 4}$ \\
\hline $\mathrm{CD}$ at $(0.01)$ & $\mathbf{4 . 3 8}$ & $\mathbf{3 . 2 7}$ \\
\hline
\end{tabular}

* Figures in paranthesis indicates arcsine transformed values 
Table.2 Effect of vigour levels on germination percentage of onion during storage (natural ageing)

\begin{tabular}{|c|c|c|c|c|c|c|c|c|c|c|}
\hline \multirow[t]{2}{*}{ Seed lots } & \multicolumn{10}{|c|}{ Storage period (months) } \\
\hline & 1 & 2 & 3 & 4 & 5 & 6 & 7 & 8 & 9 & 10 \\
\hline $\mathrm{L}_{1}$ & $\begin{array}{c}81.00 \\
(64.15)\end{array}$ & $\begin{array}{c}76.00 \\
(60.75)\end{array}$ & $\begin{array}{l}71.75 \\
(57.83)\end{array}$ & $\begin{array}{l}63.00 \\
(52.52)\end{array}$ & $\begin{array}{c}57.75 \\
(49.39)\end{array}$ & $\begin{array}{c}51.75 \\
(45.94)\end{array}$ & $\begin{array}{c}41.50 \\
(39.99)\end{array}$ & $\begin{array}{l}35.00 \\
(36.26)\end{array}$ & $\begin{array}{c}25.75 \\
(30.42)\end{array}$ & $\begin{array}{c}18.50 \\
(25.31)\end{array}$ \\
\hline $\mathrm{L}_{2}$ & $\begin{array}{c}42.00 \\
(40.37)\end{array}$ & $\begin{array}{c}40.00 \\
(39.21)\end{array}$ & $\begin{array}{c}35.50 \\
(36.42)\end{array}$ & $\begin{array}{c}25.00 \\
(29.98)\end{array}$ & $\begin{array}{c}8.75 \\
(17.00)\end{array}$ & $\begin{array}{c}5.75 \\
(13.52)\end{array}$ & $\begin{array}{c}1.75 \\
(7.15)\end{array}$ & $\begin{array}{c}1.00 \\
(49.39)\end{array}$ & $\begin{array}{c}0.75 \\
(3.82)\end{array}$ & $\begin{array}{c}0.50 \\
(1.91)\end{array}$ \\
\hline $\mathrm{L}_{3}$ & $\begin{array}{c}81.00 \\
(64.14)\end{array}$ & $\begin{array}{c}78.00 \\
(62.03)\end{array}$ & $\begin{array}{l}76.75 \\
(61.11)\end{array}$ & $\begin{array}{c}74.75 \\
(59.78)\end{array}$ & $\begin{array}{c}67.50 \\
(55.13)\end{array}$ & $\begin{array}{c}62.75 \\
(52.32)\end{array}$ & $\begin{array}{l}60.50 \\
(50.94)\end{array}$ & $\begin{array}{c}60.75 \\
(51.14)\end{array}$ & $\begin{array}{c}57.00 \\
(49.01)\end{array}$ & $\begin{array}{c}51.50 \\
(45.75)\end{array}$ \\
\hline $\mathrm{L}_{4}$ & $\begin{array}{c}63.00 \\
(52.52)\end{array}$ & $\begin{array}{l}61.00 \\
(51.34)\end{array}$ & $\begin{array}{l}60.50 \\
(50.95)\end{array}$ & $\begin{array}{l}57.00 \\
(49.00)\end{array}$ & $\begin{array}{c}57.00 \\
(49.01)\end{array}$ & $\begin{array}{c}48.50 \\
(44.03)\end{array}$ & $\begin{array}{c}38.75 \\
(38.43)\end{array}$ & $\begin{array}{c}34.00 \\
(35.64)\end{array}$ & $\begin{array}{c}31.75 \\
(34.22)\end{array}$ & $\begin{array}{c}21.00 \\
(27.26)\end{array}$ \\
\hline $\mathrm{L}_{5}$ & $\begin{array}{c}47.50 \\
(47.28)\end{array}$ & $\begin{array}{l}49.00 \\
(44.41)\end{array}$ & $\begin{array}{l}44.50 \\
(41.73)\end{array}$ & $\begin{array}{c}38.75 \\
(38.43)\end{array}$ & $\begin{array}{c}22.50 \\
(28.18)\end{array}$ & $\begin{array}{c}15.50 \\
(23.03)\end{array}$ & $\begin{array}{c}9.75 \\
(18.04)\end{array}$ & $\begin{array}{c}8.00 \\
(16.40)\end{array}$ & $\begin{array}{c}4.50 \\
(11.89)\end{array}$ & $\begin{array}{c}1.00 \\
(5.74)\end{array}$ \\
\hline $\mathrm{L}_{6}$ & $\begin{array}{c}70.00 \\
(56.81)\end{array}$ & $\begin{array}{c}68.00 \\
(55.53)\end{array}$ & $\begin{array}{l}66.00 \\
(54.32)\end{array}$ & $\begin{array}{c}51.00 \\
(45.55)\end{array}$ & $\begin{array}{c}51.00 \\
(45.55)\end{array}$ & $\begin{array}{c}43.50 \\
(41.15)\end{array}$ & $\begin{array}{c}38.50 \\
(38.23)\end{array}$ & $\begin{array}{c}31.00 \\
(33.82)\end{array}$ & $\begin{array}{c}28.00 \\
(31.93)\end{array}$ & $\begin{array}{c}17.75 \\
(24.83)\end{array}$ \\
\hline $\mathrm{L}_{7}$ & $\begin{array}{c}65.00 \\
(53.71)\end{array}$ & $\begin{array}{c}62.00 \\
(51.93)\end{array}$ & $\begin{array}{l}60.50 \\
(50.95)\end{array}$ & $\begin{array}{c}50.75 \\
(45.36)\end{array}$ & $\begin{array}{c}41.00 \\
(39.80)\end{array}$ & $\begin{array}{c}35.50 \\
(36.45)\end{array}$ & $\begin{array}{l}29.50 \\
(32.77)\end{array}$ & $\begin{array}{c}31.75 \\
(34.22)\end{array}$ & $\begin{array}{c}25.50 \\
(30.20)\end{array}$ & $\begin{array}{c}15.75 \\
(23.29)\end{array}$ \\
\hline $\mathrm{L}_{8}$ & $\begin{array}{c}61.50 \\
(51.54)\end{array}$ & $\begin{array}{c}60.00 \\
(50.76)\end{array}$ & $\begin{array}{c}58.75 \\
(49.98)\end{array}$ & $\begin{array}{c}54.00 \\
(48.43)\end{array}$ & $\begin{array}{c}49.50 \\
(44.60)\end{array}$ & $\begin{array}{c}38.50 \\
(38.24)\end{array}$ & $\begin{array}{c}31.00 \\
(33.88)\end{array}$ & $\begin{array}{c}31.00 \\
(33.80)\end{array}$ & $\begin{array}{c}26.00 \\
(30.63)\end{array}$ & $\begin{array}{c}16.75 \\
(24.07)\end{array}$ \\
\hline $\mathrm{L}_{9}$ & $\begin{array}{c}70.00 \\
(56.82)\end{array}$ & $\begin{array}{c}65.00 \\
(53.72)\end{array}$ & $\begin{array}{l}61.50 \\
(51.54)\end{array}$ & $\begin{array}{l}55.00 \\
(47.85)\end{array}$ & $\begin{array}{c}48.00 \\
(43.84)\end{array}$ & $\begin{array}{c}42.75 \\
(40.76)\end{array}$ & $\begin{array}{l}41.50 \\
(39.99)\end{array}$ & $\begin{array}{l}39.00 \\
(38.62)\end{array}$ & $\begin{array}{c}33.00 \\
(35.65)\end{array}$ & $\begin{array}{c}25.00 \\
(29.97)\end{array}$ \\
\hline $\mathrm{L}_{10}$ & $\begin{array}{c}71.75 \\
(58.26)\end{array}$ & $\begin{array}{c}70.00 \\
(56.82)\end{array}$ & $\begin{array}{c}68.50 \\
(55.75)\end{array}$ & $\begin{array}{c}57.75 \\
(49.39)\end{array}$ & $\begin{array}{c}54.00 \\
(47.28)\end{array}$ & $\begin{array}{c}46.75 \\
(43.07)\end{array}$ & $\begin{array}{c}43.50 \\
(41.15)\end{array}$ & $\begin{array}{c}41.75 \\
(40.19)\end{array}$ & $\begin{array}{c}38.00 \\
(38.04)\end{array}$ & $\begin{array}{c}27.75 \\
(31.72)\end{array}$ \\
\hline $\mathrm{L}_{11}$ & $\begin{array}{c}87.00 \\
(68.88)\end{array}$ & $\begin{array}{c}84.50 \\
(66.70)\end{array}$ & $\begin{array}{l}81.00 \\
(64.14)\end{array}$ & $\begin{array}{c}74.00 \\
(59.50)\end{array}$ & $\begin{array}{c}71.75 \\
(57.83)\end{array}$ & $\begin{array}{c}72.00 \\
(58.04)\end{array}$ & $\begin{array}{l}64.75 \\
(53.54)\end{array}$ & $\begin{array}{l}61.00 \\
(51.34)\end{array}$ & $\begin{array}{c}61.00 \\
(51.34)\end{array}$ & $\begin{array}{c}64.75 \\
(53.52)\end{array}$ \\
\hline $\mathrm{L}_{12}$ & $\begin{array}{c}85.75 \\
(67.74)\end{array}$ & $\begin{array}{c}86.00 \\
(68.03)\end{array}$ & $\begin{array}{c}83.50 \\
(65.89)\end{array}$ & $\begin{array}{c}76.50 \\
(60.88)\end{array}$ & $\begin{array}{c}72.50 \\
(58.28)\end{array}$ & $\begin{array}{c}69.00 \\
(56.16)\end{array}$ & $\begin{array}{c}61.00 \\
(51.34)\end{array}$ & $\begin{array}{l}57.75 \\
(49.39)\end{array}$ & $\begin{array}{c}52.50 \\
(46.32)\end{array}$ & $\begin{array}{c}45.00 \\
(42.11)\end{array}$ \\
\hline $\mathrm{L}_{13}$ & $\begin{array}{c}91.00 \\
(72.58)\end{array}$ & $\begin{array}{c}90.00 \\
(71.75)\end{array}$ & $\begin{array}{c}89.75 \\
(71.30)\end{array}$ & $\begin{array}{c}87.50 \\
(69.19)\end{array}$ & $\begin{array}{c}85.00 \\
(67.24)\end{array}$ & $\begin{array}{c}85.00 \\
(67.22)\end{array}$ & $\begin{array}{c}81.75 \\
(64.65)\end{array}$ & $\begin{array}{c}82.00 \\
(64.88)\end{array}$ & $\begin{array}{c}75.50 \\
(60.22)\end{array}$ & $\begin{array}{c}75.00 \\
(59.99)\end{array}$ \\
\hline $\mathrm{L}_{14}$ & $\begin{array}{l}33.50 \\
(35.24)\end{array}$ & $\begin{array}{c}30.00 \\
(33.19)\end{array}$ & $\begin{array}{l}26.00 \\
(30.63)\end{array}$ & $\begin{array}{c}14.50 \\
(22.22)\end{array}$ & $\begin{array}{c}2.50 \\
(8.74)\end{array}$ & $\begin{array}{c}2.00 \\
(7.94)\end{array}$ & $\begin{array}{c}0.75 \\
(3.82)\end{array}$ & $\begin{array}{c}0.50 \\
(1.91)\end{array}$ & $\begin{array}{c}0.50 \\
(1.91)\end{array}$ & $\begin{array}{c}0.00 \\
(0.00)\end{array}$ \\
\hline $\mathrm{L}_{15}$ & $\begin{array}{l}54.00 \\
(47.28)\end{array}$ & $\begin{array}{l}52.00 \\
(46.13)\end{array}$ & $\begin{array}{c}52.00 \\
(46.13)\end{array}$ & $\begin{array}{c}40.50 \\
(39.41)\end{array}$ & $\begin{array}{c}25.50 \\
(30.20)\end{array}$ & $\begin{array}{c}21.00 \\
(27.25)\end{array}$ & $\begin{array}{l}13.75 \\
(21.68)\end{array}$ & $\begin{array}{c}11.00 \\
(19.31)\end{array}$ & $\begin{array}{c}5.50 \\
(13.26)\end{array}$ & $\begin{array}{c}2.00 \\
(7.94)\end{array}$ \\
\hline Mean & $\begin{array}{c}66.93 \\
(55.60)\end{array}$ & $\begin{array}{c}64.76 \\
(54.14)\end{array}$ & $\begin{array}{l}62.50 \\
(52.58)\end{array}$ & $\begin{array}{c}\mathbf{5 4 . 7 3} \\
(\mathbf{4 7 . 8 2})\end{array}$ & $\begin{array}{c}47.53 \\
(42.80)\end{array}$ & $\begin{array}{c}42.60 \\
(39.75)\end{array}$ & $\begin{array}{l}\text { 37.11 } \\
(\mathbf{3 5 . 7 0})\end{array}$ & $\begin{array}{l}\text { 35.00 } \\
(\mathbf{3 4 . 1 0})\end{array}$ & $\begin{array}{c}\text { 31.00 } \\
(\mathbf{3 1 . 2 6})\end{array}$ & $\begin{array}{c}25.42 \\
(26.89)\end{array}$ \\
\hline S.Em. \pm & 1.05 & 1.09 & 0.99 & 0.80 & 0.79 & 0.94 & 1.07 & 1.06 & 1.02 & 0.82 \\
\hline $\mathrm{CD}$ at $(0.01)$ & 4.10 & 4.23 & 3.85 & 3.11 & 3.08 & 3.66 & 4.17 & 4.10 & 3.95 & 3.18 \\
\hline
\end{tabular}

* Figures in paranthesis indicates arcsine transformed values 
Table.3 Correlation among the vigour tests at the initiation of storage period

\begin{tabular}{|c|c|c|c|c|c|c|}
\hline & $\begin{array}{c}\text { Germination } \\
(\%)\end{array}$ & $\begin{array}{c}\text { Field } \\
\text { emergence } \\
(\%)\end{array}$ & $\begin{array}{c}\text { Electrical } \\
\text { conductivity } \\
\left(\mathbf{d S m}^{-1}\right)\end{array}$ & $\begin{array}{l}\text { Seedling } \\
\text { vigour } \\
\text { index-I } \\
\text { (SVI-I) }\end{array}$ & $\begin{array}{c}\text { Germination } \\
\text { after } \\
\text { accelerated } \\
\text { ageing }(\%)\end{array}$ & $\begin{array}{c}\text { Mean } \\
\text { germination } \\
\text { time }(\mathbf{h})\end{array}$ \\
\hline Germination (\%) & 1 & & & & & \\
\hline Field emergence $(\%)$ & $0.949 * *$ & 1 & & & & \\
\hline Electrical conductivity $\left(\mathrm{dSm}^{-1}\right)$ & $-0.866 * *$ & $-0.906 * *$ & 1 & & & \\
\hline Seedling vigour index-I (SVI-I) & $0.988 * *$ & $0.972 * *$ & $-0.908 * *$ & 1 & & \\
\hline Germination after accelerated ageing (\%) & $0.923 * *$ & $0.925 * *$ & $-0.933 * *$ & $0.955 * *$ & 1 & \\
\hline Mean germination time (h) & $-0.852 * *$ & $-0.900 * *$ & $0.872 * *$ & $-0.896 * *$ & $-0.857 * *$ & 1 \\
\hline
\end{tabular}

** Correlation is significant at the 0.01 level of probability 
Fig.1 Graphical representation of germination and germination after accelerated ageing

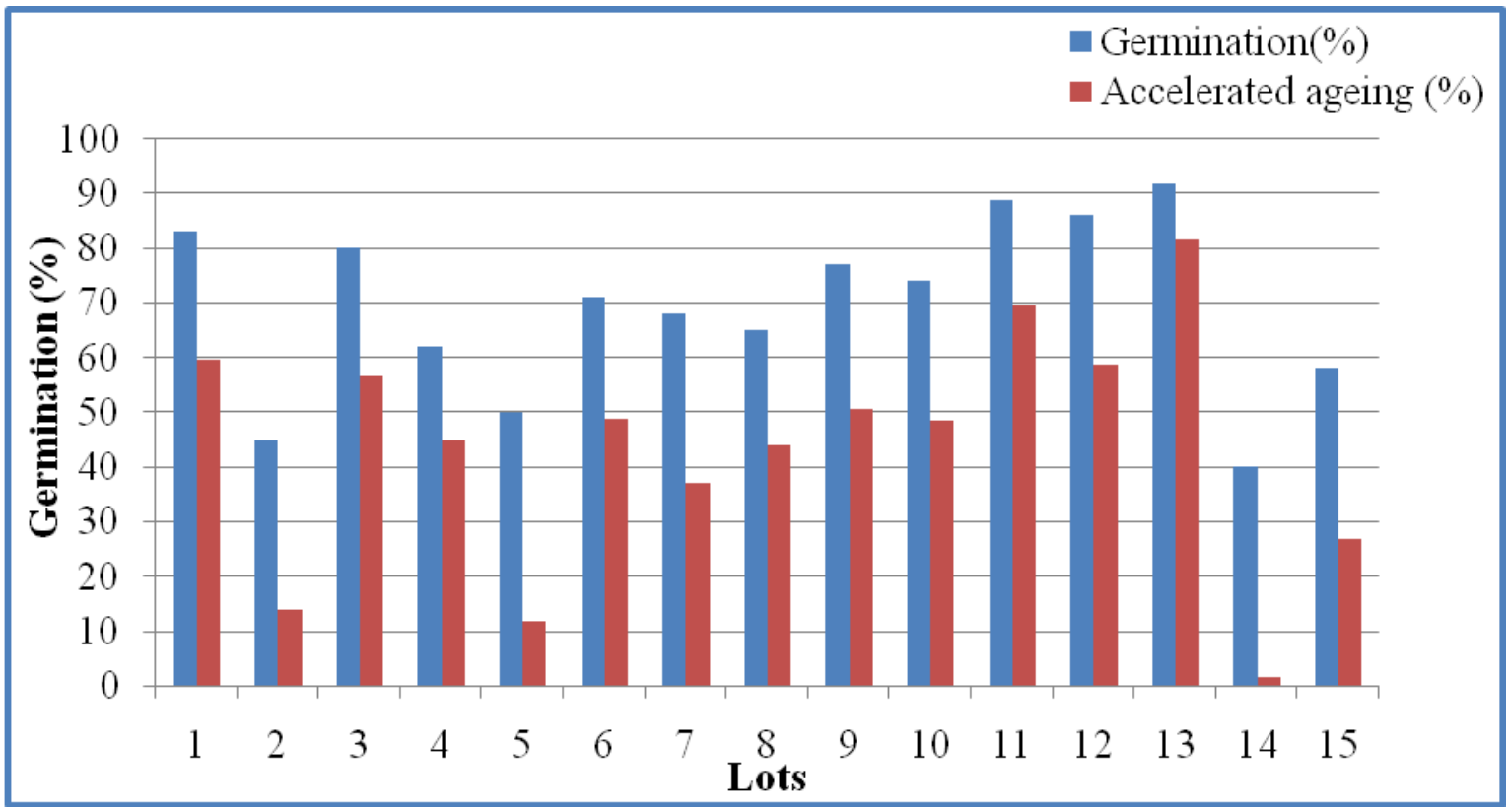

Fig.2 Relationship between germination and germination after accelerated ageing

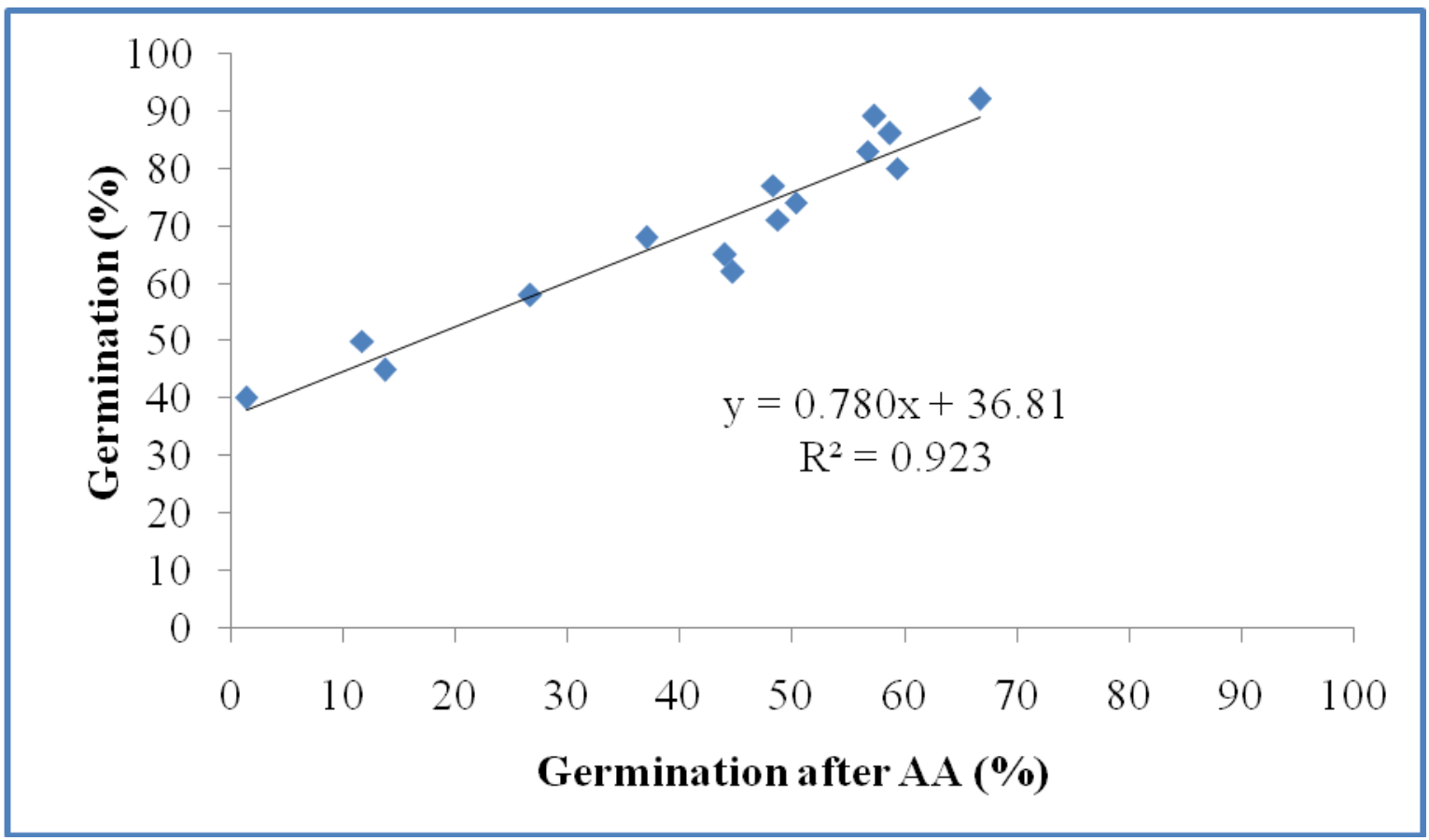


Fig.3 Relationship between field emergence and germination after accelerated ageing

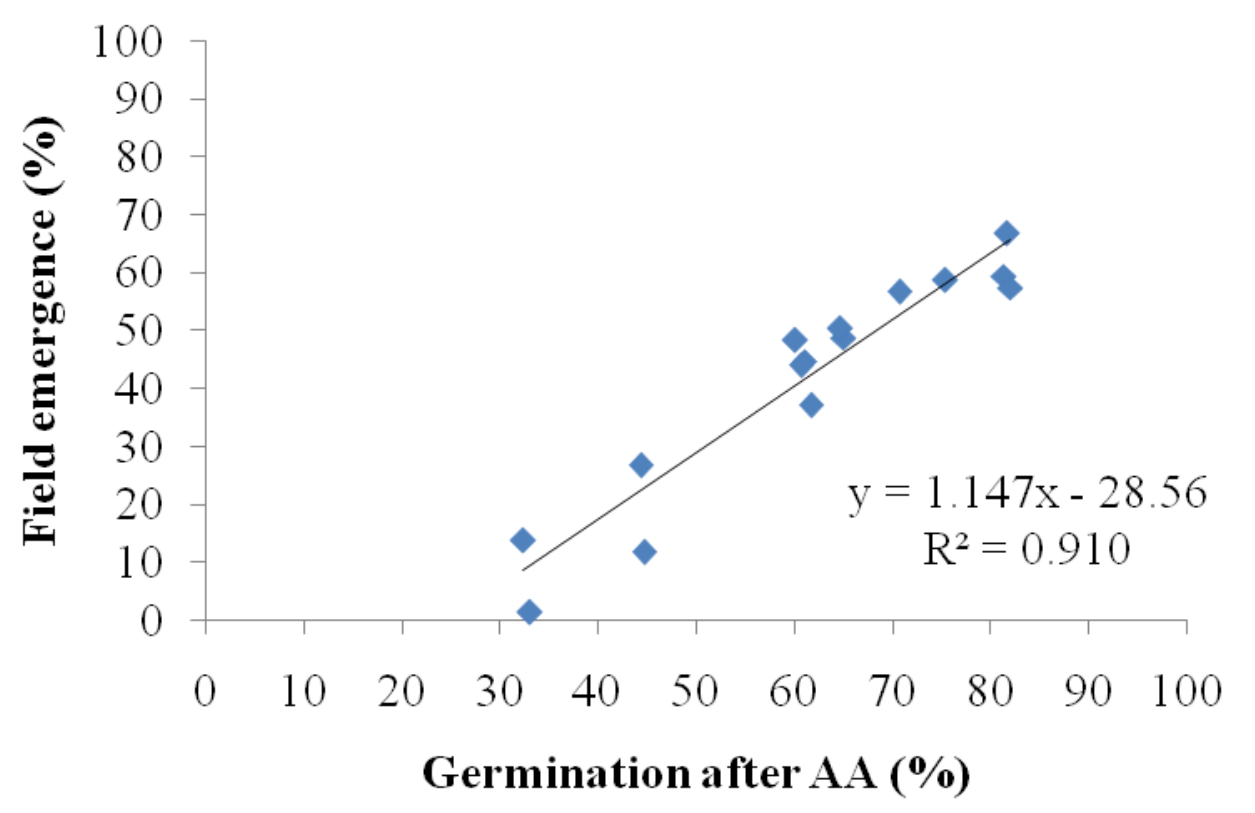

Fig.4 Relationship between electrical conductivity and germination after accelerated ageing

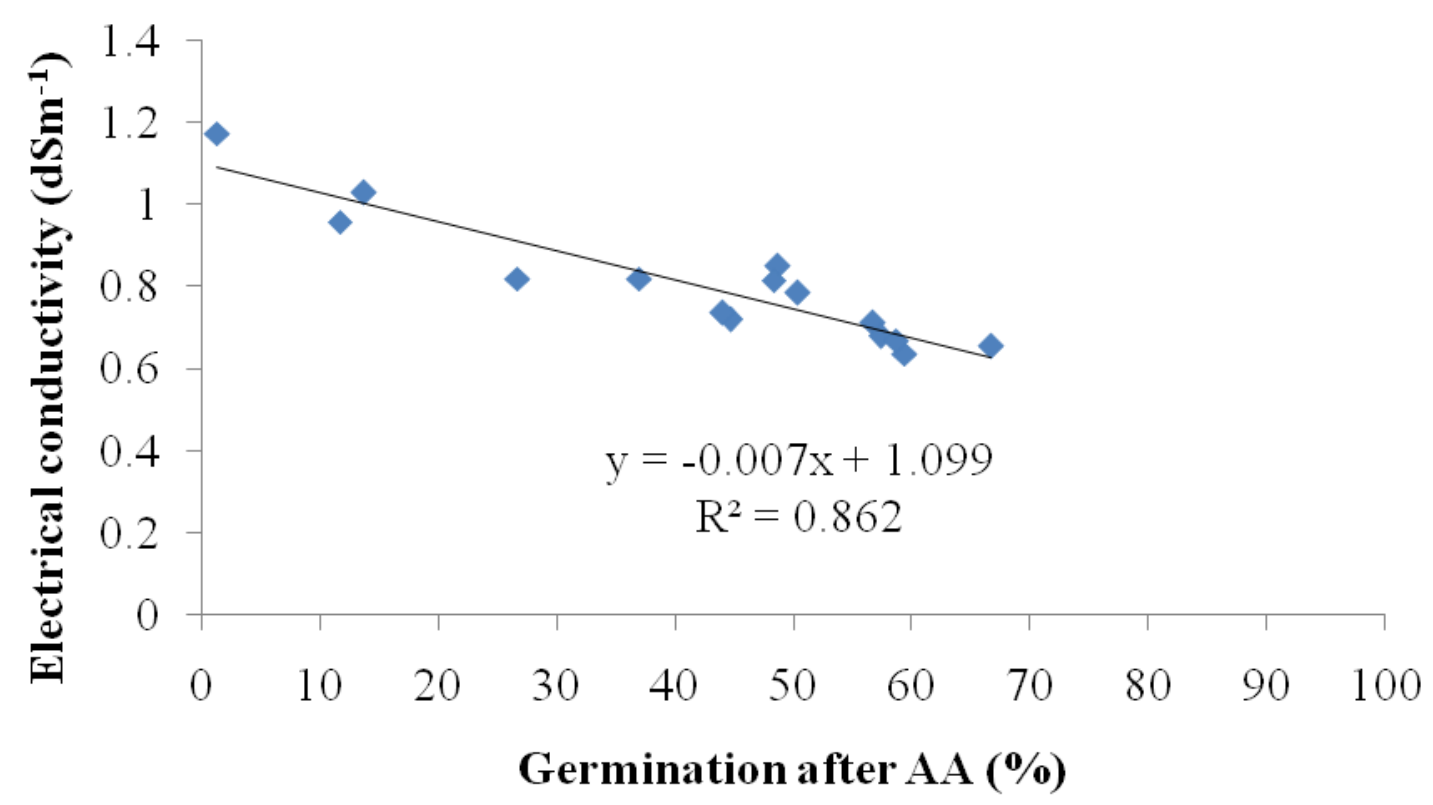


Fig.5 Relationship between mean germination time and germination after accelerated ageing

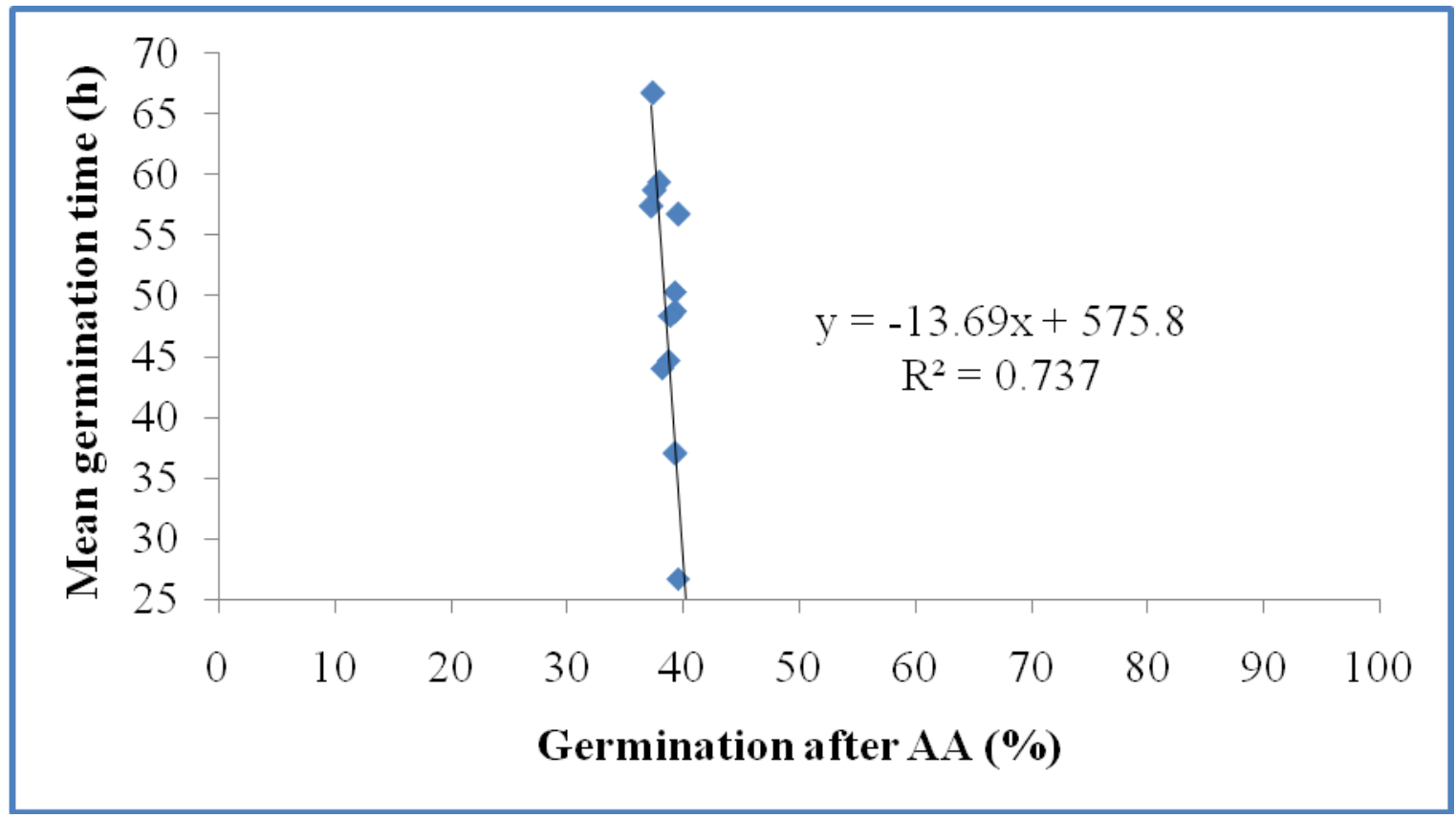

\section{Acknowledgments}

The authors are thankful to the Department of Seed Science and technology, University of Agricultural Sciences, Dharwad.

\section{References}

Anon., 2013, International Rules for Seed Testing. Seed Sci. Techol., 29: 37-41.

Ashraf, A. and Habib, M., 2011, Ash (Fraxinus excelsior) seed quality in relation to seed deterioration under accelerated ageing conditions. African J. Biotechnol., 10(36): 6961-6972.

Delouche, J. C. and Baskin, C. C., 1973, Accelerated ageing techniques for predicting the relative storability of seed lots. Seed Sci. Technol., 1: 427452.

Doijode, S. D., 1998, Conservation of Allium сера germplasm in India. National symposium on vegetables, Varanasi, India, Abstract, 3(2): 168-210.

Doijode, S. D., 2001, Bulb crops. In: seed storage of horticultural crops. 1st. New York, USA: The Haworth Press, Inc., pp. 189-210.

Horbowicz, M., 1997, Changes of carbohydrate contents during natural and accelerated ageing of some vegetable seeds. Curr. Plant Sci. Biotechnol. Agric., 30: 803-808.

Kataki, P. K., Horbowicz, M., Taylor, A. G. and Obendorf, R. L., 1997, Changes in sucrose, cyclitols and their galactosyl derivatives with seed ageing. Curr. Plant Sci. Biotechnol. Agric., 30: 515522.

Khan, M. M., Iqbal, M. J., Abbas, M. and Usman, M., 2003, Effect of ageing on viability, vigour and chromosomal damage in pea (Pisum sativum L.) seeds. Pak. J. Agri. Sci., 40: 1-2.

Maskri, A. Y., Khan, M., Iqbal, J. M. and Abbas, M., 2004, Germinability, vigour and electrical conductivity changes in accelerated aged watermelon (Citrullus lanatus T.) seeds. J. Food Agric. Environ., 2(3): 
100-103.

McDonald, M. B. 1995, Standardization of seed vigour tests. Seed vigour testing (ed. H.A. van de Venter), 88-97, International Seed Testing Association, Zurich.

Mosavi, N. S. M., Gholami, H., Kord, G. H., Sadeghi, M. and Sedighi, E., 2011, Free fatty acid and electrical conductivity changes in cotton seed (Gossypium hirsutum L.). Int. J. Agric. Sci., 1(2): 62-66.

Panse, V. G. and Sukhatme, P. V., 1985, Statistical methods for agricultural workers, ICAR, New Delhi, pp. 327340.

Sundararaj, N., Nagaraju, S., Venkataramu, M. N and Jagannath, M. K., 1972, Design and analysis of experiment, Uni. Agri. Sci., Bangalore, Karnataka, India.

Yousof, F. I., Ward, A., Ibrahim, M. S. and Dahab, A., 2016, Efficiency of some seed vigour tests for field emergence prediction of onion seed. J. Plant Product. Mansoura Univ., 7(11): 1173-1178.

\section{How to cite this article:}

Manjunath V. Balikai, N. K. Biradarpatil, Jagadish Hosamani and Biradar, M. S. 2020. Accelerated Ageing for Prediction of Storability in Onion (Allium cepa L.) Seeds. Int.J.Curr.Microbiol.App.Sci. 9(03): 3163-3172. doi: https://doi.org/10.20546/ijcmas.2020.903.362 